\title{
Filigrane
}

Écoutes psychanalytiques

\section{Les paradoxes et défis de la transmission de la psychanalyse. Quelques enjeux identitaires dans le processus de transmission}

\section{Gabriela Legorreta}

Volume 27, numéro 1, 2018

La transmission de la psychanalyse

URI : https://id.erudit.org/iderudit/1055603ar

DOI : https://doi.org/10.7202/1055603ar

Aller au sommaire du numéro

Éditeur(s)

Revue Santé mentale au Québec

ISSN

1192-1412 (imprimé)

1911-4656 (numérique)

Découvrir la revue

Citer cet article

Legorreta, G. (2018). Les paradoxes et défis de la transmission de la psychanalyse. Quelques enjeux identitaires dans le processus de transmission. Filigrane, 27(1), 61-77. https://doi.org/10.7202/1055603ar

\section{Résumé de l'article}

Ce texte aborde le thème de la transmission de la psychanalyse à partir de l'exploration des paradoxes et défis inhérents à cette transmission. Il aborde également certains enjeux identitaires dans le processus de transmission. Après avoir clarifié ce que la psychanalyse vise à transmettre, l'auteur propose trois voies de la transmission. Une première est l'expérience analytique. Une deuxième est l'expérience de la théorie. La troisième voie est l'expérience de supervision. C'est dans cette section que l'auteur décrit les divers défis qui guettent le processus de transmission, autant pour le superviseur que pour le supervisé, en commençant par la création d'un espace élaboratif dans le cadre de la supervision. Cet espace a un potentiel régressif qui s’active dans le processus de supervision; ce qui peut favoriser un relâchement des repères identitaires qui demande de tolérer les limites floues où la stabilité de l'identité est parfois menacée chez les deux protagonistes engagés dans le processus de transmission. Appuyé par des vignettes cliniques, le texte aborde également le danger de l'idéalisation dans la supervision ainsi que le thème de

l'entrecroisement de la supervision et de l'analyse personnelle. L'auteur propose enfin certaines façons de corroborer les traces de la transmission. 


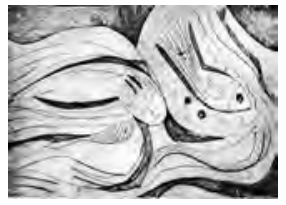

\title{
Les paradoxes et défis de la transmission de la psychanalyse. Quelques enjeux identitaires dans le processus de transmission
}

\author{
Gabriela Legorreta
}

\begin{abstract}
Résumé: Ce texte aborde le thème de la transmission de la psychanalyse à partir de l'exploration des paradoxes et défis inhérents à cette transmission. Il aborde également certains enjeux identitaires dans le processus de transmission. Après avoir clarifié ce que la psychanalyse vise à transmettre, l'auteur propose trois voies de la transmission. Une première est l'expérience analytique. Une deuxième est l'expérience de la théorie. La troisième voie est l'expérience de supervision. C'est dans cette section que l'auteur décrit les divers défis qui guettent le processus de transmission, autant pour le superviseur que pour le supervisé, en commençant par la création d'un espace élaboratif dans le cadre de la supervision. Cet espace a un potentiel régressif qui s'active dans le processus de supervision; ce qui peut favoriser un relâchement des repères identitaires qui demande de tolérer les limites floues où la stabilité de l'identité est parfois menacée chez les deux protagonistes engagés dans le processus de transmission. Appuyé par des vignettes cliniques, le texte aborde également le danger de l'idéalisation dans la supervision ainsi que le thème de l'entrecroisement de la supervision et de l'analyse personnelle. L'auteur propose enfin certaines façons de corroborer les traces de la transmission.
\end{abstract}

Mots clés: transmission; enjeux identitaires; supervision; psychanalyse personnelle; théorie psychanalytique.

\begin{abstract}
This paper adresses the challenges and paradoxes inherent to the transmission of psychoanalysis and considers some of the identification issues within the transmission process itself. First clarifying what is being sought to transmit, the author then presents three channels of transmission: the analytical experience; the theoretical experience; the supervisory experience. The latter is discussed in terms of specific challenges for both supervisor and supervisee, notably through the creation of a space conducive to elaboration. The regressive allowed by such a space demands of both protagonists a tolerance for a measure of instability affecting the boundaries of identity. Clinical vignettes illustrate these challenges. The paper also deals with the risks of idealization pertaining to the supervision process and the enmeshment of the latter with personal analysis.
\end{abstract}


Key Words: transmission; identity issues; supervision; personal analysis; psychoanalytic theory.

\begin{abstract}
$\Lambda$ border le thème de la transmission et son lien avec des enjeux identitaires est un processus stimulant et générateur de questionnements. Pendant la préparation de ce texte, chaque réflexion venait accompagnée de nouvelles questions importantes: De quelle façon mon identité d'analyste s'est-elle forgée et continue-t-elle à se forger? La formation institutionnelle a-t-elle permis ou entravé la transmission? Qui ont été les personnes et les groupes qui ont joué et qui continuent de jouer un rôle important dans la transmission? Cette réflexion a eu un effet transformatif: ma vision de la transmission s'est enrichie, ce qui, je l'espère, contribuera à mon enthousiasme et à ma volonté de transmettre à travers ce texte.

Nous allons commencer par une réflexion sur ce que l'on transmet pour ensuite aborder le sujet des voies de la transmission.
\end{abstract}

\title{
I. Que transmet-on?
}

On transmet un courage. Le courage de prendre une position d'ouverture à une expérience émotionnelle transformative. Une position audacieuse qui sollicite un relâchement des repères identitaires; qui demande de tolérer un état psychique aux limites floues où la stabilité de l'identité est parfois menacée chez les deux protagonistes engagés dans le processus de transmission.

On transmet un esprit, un esprit analytique, un type de réceptivité, de disponibilité. Une réceptivité envers soi-même, envers ceux qui décident de s'engager dans un processus analytique ou de supervision, et également, envers la réalité dans ses multiples dimensions sociales et culturelles qui ne cessent de nous interpeller.

L'esprit psychanalytique ne s'enseigne pas, cette réceptivité ne peut s'obtenir que par l'expérience, par l'effort assidu pour soutenir cette curiosité, cette ouverture, cette perméabilité envers soi-même et envers l'autre. C'est lorsqu'on est saisi par la force des processus inconscients, quand on est "touché au vif», que l'on constate jusqu'à quel point, comme dirait Freud, nous ne sommes pas maitres dans notre propre maison. Cette expérience, aussi déstabilisante soit-elle, élargit notre regard et notre disposition envers nous-mêmes et envers l'autre. Elle amplifie notre sensibilité et notre capacité d'accueillir l'inconscient de l'autre. A. Lussier (1992, p. 487) nous l'a bien dit: «Notre identité en tant qu'analyste n'émane pas d'un “savoir" et ne 
procède pas d'une accumulation de connaissances. C'est à partir de l'analyse personnelle et de la communication avec son propre inconscient qu'on devient analyste.»

Cette réceptivité demande d'aller toujours contre la tendance du moi à assimiler toute expérience à ce qui est familier. Elle exige l'introduction d'un écart de temps pour résister à la tentation de tout réduire d'avance à ce que l'on croit comprendre, pour renoncer temporairement à une position de «savoir», pour se défaire de la tentation d'agir en vue de trouver un soulagement. C'est un travail psychique qui exige simultanément quelque chose de passif et d'actif; une sorte de tension qui faciliterait l'accès à une expérience psychique particulière. Cette expérience permettra de développer et de tolérer ce que de Michel de M’Uzan (2015) a décrit comme «une inquiétude permanente»-j'y reviendrai - dans laquelle on est appelé à rester dans le suspense pour permettre que quelque chose nous surprenne. Comme Réjean Ducharme l'a affirmé: «pour vraiment vivre il faut être sur le qui-vive».

En suivant cette ligne de réflexion, Dominique Scarfone (2014) propose la notion de "passibilité », qui aborde aussi bien la question de la réceptivité que de l'identité. Ce terme se réfère à une réceptivité de l'infans, expérience humaine qui ne peut être captée par le langage. Scarfone affirme que pour atteindre l'état de "passibilité », «il faut faire taire le discours incessant du moi qui cherche à nommer, classer, évaluer, interpréter, au lieu de laisser passer quelque chose, de se laisser traverser, voire transpercer». Ceci permet à l'analyste d' "accueillir un transfert de ce qui ne saurait passer par les seuls canaux du discours» (p. 1360-1361). Il s'agit donc d'une disposition qui nous fera inévitablement rencontrer les zones de résistance porteuses d'un potentiel paradoxal: en se posant comme l'obstacle de l'analyse, elle nous signale en même temps le chemin à suivre.

On pense ici d'ailleurs à Pontalis (1997) et à sa "pensée rêvante ", à savoir une attention flottante, mobile. On pense aussi aux recommandations de Freud qui nous prévient de ne pas confondre les rêves et les contenus manifestes, en proposant au clinicien d'adopter une attention en "égal suspens» qui saura déceler les déformations, condensations, et déplacements à travers les productions langagières.

Bion abordera la question de la réceptivité et de la disponibilité en avançant la notion de "capacité négative», c'est-à-dire la capacité de tolérer la douleur et la confusion associée à l'état d'ignorance. Bion (1970) recommande d'approcher la rencontre avec le patient sans mémoire ni désir afin de laisser un espace vide. C'est dans l'espace du moment présent dans 
l'expérience unique du lien entre thérapeute et patient qu'une nouvelle pensée peut surgir. La notion de capacité négative chez Bion a ses origines dans ses observations de l'importance de la mère à tolérer l'expérience souvent troublante qui est le résultat des identifications projectives de son petit enfant. La mère utilisera sa capacité négative et sa capacité de rêverie afin de transformer l'expérience afin qu'elle soit tolérable pour le petit enfant. Il est intéressant de constater que Freud établissait à cet égard déjà des lignes directrices pour les futurs analystes en indiquant que «les meilleurs résultats thérapeutiques [...] s'obtiennent lorsque l'analyste procède sans s'être préalablement tracé de plan, se laisse surprendre par tout fait inattendu, conserve une attitude détachée et évite toute idée préconçue [...]. Il devra passer, suivant les besoins, d'une attitude psychique à une autre, éviter toute spéculation, toute rumination mentale» (Freud, 1923, p. 65) On voit ici les origines de ce que Bion a plus tard décrit et développé sous le concept de capacité négative. Pour ce faire, Bion a par ailleurs souvent utilisé une citation de Maurice Blanchot (1969): «La réponse est le malheur de la question [... - la réponse est le malheur ou la maladie de la curiosité - elle la tue».

\section{Les voies de la transmission}

\section{L'expérience analytique}

Une voie «royale» de la transmission est sans doute l'expérience de l'analyse personnelle. L'esprit analytique a son origine dans l'intuition personnelle de l'existence de quelque chose d'insaisissable qui nous habite, qui nous échappe et qui nous amène sans trop le savoir à une recherche de soi dans l'écoute de l'autre en soi, par le biais de l'invitation à la parole libre. Cette «traversée» comme dit Pontalis (Pontalis, 1997) qui est l'expérience analytique, mettra en mouvement un parcours qui ne cessera de nous surprendre, ne serait-ce que par la réalisation des forces qui résistent à donner accès au monde intérieur de l'inconscient. Elle élargit la réceptivité envers autrui, ainsi qu'envers les différents secteurs du psychisme. La psyché s'enrichit, se flexibilise, élargissant son potentiel de penser et d'éprouver ce qui était impensable. Les deux protagonistes de l'analyse auront la récompense de leur travail ardu quand ils éprouveront le plaisir qui accompagne des moments charnières où quelque chose du domaine de l'inconscient arrive à se transformer et devient domaine du psychisme. L'expérience analytique transmettra cet esprit et cette capacité négative. 


\section{La place et l'expérience de la théorie}

J'évoque l'expérience de la théorie puisque s'immerger sérieusement dans l'étude d'un texte a toujours eu pour moi un impact émotionnel marquant. Lire et étudier sérieusement un auteur est une expérience émotionnelle. Une réceptivité marquée par l'esprit analytique envers les textes théoriques permettra des expériences émotionnelles diverses. Le processus de transmission requiert une capacité de questionner, de dialoguer, de redécouvrir l'importance d'un texte. L'approche que nous souhaitons avoir de la théorie psychanalytique veut qu'elle soit directement guidée sur une pratique clinique, autrement dit, que notre rapport aux textes théoriques suscite un constant questionnement à partir des questions qui surgissent de la pratique clinique. Seule une telle attitude permettra d'acquérir progressivement une connaissance solide des piliers de la théorie psychanalytique. Un de ces piliers est sans doute la métapsychologie freudienne. Même si on est novice dans la connaissance de la théorie, il est important de tenter progressivement de poser le plus clairement possible des repères qui nous donnent un appui sur notre intervention clinique.

Le recours à la théorie permet aussi de saisir et d'intégrer la richesse de la méthode analytique (dont les éléments essentiels sont le cadre, l'association libre, l'attention flottante). Elle permet ainsi de comprendre l'importance de l'offrir et de l'utiliser avec rigueur. Ce n'est qu'une forte conviction en sa puissance qui permettra à l'analyste de vaincre ses résistances à la mettre de l'avant et à communiquer et transmettre sa valeur. L'analyse personnelle, la supervision, les séminaires, les discussions cliniques, contribueront à rendre la théorie vivante.

\section{Une autre voie de la transmission:}

\section{I'expérience de supervision. Les multiples défis et rôles dans l'espace de supervision: des enjeux identitaires}

L'espace de supervision est complexe et exigeant. Il exige des deux participants des dispositions variables tant intellectuelles qu'émotionnelles. Le superviseur doit avoir une flexibilité dans son écoute et décider à quoi il doit donner la priorité, soit la scène psychique du patient (repérer le transfert et les zones de résistance), soit la scène hic et nunc de ce qui se passe entre le superviseur et le supervisé, ou encore le souci de transmettre un «savoir-faire, voire une technique, des aspects clinico-théoriques du matériel rapporté». L'idéal serait de tenir compte de toutes ses dimensions et de privilégier ce qui favorisera l'évolution du supervisé. 
Nous souhaitons proposer une réflexion sur ces défis, en commençant par ce qui suit.

\section{a) La création d'un espace élaboratif en supervision: un accueil analytique}

Un des défis les plus importants de la transmission de la psychanalyse est la création d'un espace élaboratif. Il s'agit d'un défi de taille, car il faut tenir compte du fait que cet espace est traversé par des forces pulsionnelles provenant de plusieurs sources qui affectent les deux protagonistes. Le patient qui est "présenté» en supervision est une sorte de fiction construite par le supervisé; une fiction chargée de forces pulsionnelles. Comment mettre sur pied un espace de jeu dans un champ de forces qui combine la scène transférentielle inconsciente du supervisé, de son patient, ainsi que celle qui s'installe dans le rapport entre superviseur et supervisé?

Ces questions nous amènent à considérer que les conditions propices à l'émergence d'un espace élaboratif en supervision supposent le même type de disposition dans le couple superviseur-supervisé que dans le couple analyste-analysé (réceptivité, absence de préconception). La supervision demande un constant renouvellement de la conviction de l'identité d'analyste. Le danger est toujours de se réfugier dans une position de «savoir» plutôt que de se laisser aller avec l'expérience proposée par le supervisé.

La théorie du champ est utile dans la réflexion sur cette question. Pichon Rivière a introduit cette théorie et elle a été développée par d'autres analystes contemporains dont Baranger (1979), Brown (2011), Ferro et Civitarese (2014) et Ogden (1994). La théorie du champ analytique propose que la méthode analytique, et j'ajouterais le travail de supervision, invite les deux participants à suspendre la fonction inhibitrice du moi et à se laisser aller dans un espace régressif. Dans cet espace, un champ de forces s'installe. Ces forces construisent, à l'insu de l'analyste et de l'analysant, une sorte d'expérience unique à ce couple particulier.

Quels sont donc les éléments qui peuvent contribuer à la création de cet espace élaboratif? Un élément essentiel est l'attitude et la position du superviseur qui tente de libérer son écoute des processus secondaires afin de s'ouvrir à l'écoute d'une écoute (Faimberg, 2005), c'est-à-dire que le superviseur n'écoute pas seulement la narration du supervisé, mais qu'il essaye de saisir, entre autres choses, la façon dont il écoute son patient, ce qu'il écoute et ce qu'il n'écoute pas. 
Même s'il faut tenir compte de l'importance de la transmission de la théorie, comme nous l'avons formulé plus tôt, l'écoute ne doit pas se centrer, comme le souligne Jean-Paul Valabrega (1976), sur la transmission d'un savoir. Les échanges doivent encourager une véritable dimension de recherche partagée.

Peut-on penser à un cadre pour la supervision? Nous proposons que, de la même façon que l'on installe un «cadre» dans l'espace analytique, la supervision en requiert un également. Thomas Ogden (2005) nous rappelle que la supervision analytique exige les mêmes libertés et protections que la relation analytique. Le superviseur est chargé de créer un cadre qui garantit la liberté pour le supervisé de penser et d'élaborer de vive voix. Le cadre de la supervision doit offrir un sentiment de sécurité, lui permettre d'avoir confiance dans le fait que ses efforts pour être honnête seront respectés.

Une attitude de recherche partagée, l'établissement d'un cadre et une confiance dans la fonction tiercéisante feront en sorte que le supervisé sera disposé à mettre en jeu et en circulation ce qui lui pose problème. Il pourra ainsi développer une curiosité par ses propres mécanismes internes dans l'expérience toujours singulière du travail analytique avec son patient. Il s'agit donc d'encourager le supervisé à ne pas aborder le matériel clinique à partir de "la bonne interprétation", conçue pour corroborer ses propres théories mais plutôt en se laissant écouter ce qui lui paraît moins familier. Comment encourageons-nous le supervisé à s'approcher de cette position? Il faut d'abord souligner l'importance d'adopter une attitude d'humilité et de réserve qui, en s'éloignant de la position du sujet «supposésavoir» - même si celle-ci répondrait au désir du supervisé - lui permettra de s'identifier à un accueil de l'imprévu et de s'affranchir du besoin de contrôle. L'esprit analytique, ou la capacité négative, est très souvent présent chez ceux qui font une demande de supervision; il s'agit de l'identifier, de la nommer, de l'amplifier et de souligner son importance fondamentale. Cet esprit analytique est une flamme qu'il faut attiser et aider à grandir. La confiance dans la valeur d'un esprit analytique (même s'il est rudimentaire) aidera le supervisé à se risquer à une pensée et une parole plus libre dans son travail clinique.

Cet espace devra permettre progressivement de lever des censures surmoïques, ce qui permettra au supervisé de considérer comme particulièrement pertinente l'évocation de certains mouvements contre-transférentiels, de les repérer (même lorsqu'ils sont agis), de les expliciter et, par leur mise en sens, de contribuer à l'élaboration transférentielle. 
Une vignette clinique semble éclairante à cet effet. Marie me présente le cas d'une femme qui développe un transfert amoureux et érotique envers elle; la patiente éprouve des excitations sexuelles en séance. Lors d'une séance particulièrement intense la patiente déclare "je vous aime». Marie répond "ça c'est une idée intéressante! ». Marie me confie la difficulté qu'elle a éprouvée à vaincre la tentation de ne pas me parler de cet échange: elle est troublée par sa réaction et a honte de son intervention. J'encourage Marie à continuer à soutenir le courage de réfléchir ce qui se passe avec sa patiente et avec moi. Notre échange ouvert a jeté de la lumière sur la charge érotique et hostile dans le transfert dont Marie se défendait à son insu. La honte ressentie envers moi reflétait la honte éprouvée par sa patiente.

\section{b) Des enjeux identitaires}

La discussion précédente sur la création d'un espace élaboratif fait allusion au potentiel de régression activé dans le processus de supervision comme dans celui de l'analyse personnelle. La parole libre en supervision et les forces pulsionnelles qui habitent son champ ont la possibilité de favoriser un relâchement des repères identitaires qui demande de tolérer un état psychique aux limites floues, où la stabilité de l'identité est parfois menacée chez les deux protagonistes engagés dans le processus de transmission.

Michel de M'Uzan s'est beaucoup intéressé à la question de l'identité. Sa notion de «vacillement identitaire» fait référence à la disposition dans laquelle doit se mettre l'analyste, c'est-à-dire un certain état de dépersonnalisation qui suscite une expérience d'«inquiétude permanente». De M’Uzan (2009, p. 97) affirme qu'elle consiste à "rejoindre ce point où doit se reconnaitre comme donnée fondamentale de l'être le caractère incertain, et même plus ou moins secrètement durable, incertain et aléatoire des frontières entre tous les ordres, au point d'en faire presque une plaidoirie, je ne cesse de le rappeler».

Si nous mentionnons certains aspects du travail de M. de M'Uzan, c'est pour illustrer le type d'expérience qui est susceptible d'avoir lieu lorsque l'analyste ouvre non seulement la porte de son cabinet, mais aussi la porte de son psychisme, y compris son expérience émotionnelle. Ceci favorise l'accès à des aspects de la vie psychique du supervisé et de son patient qui n'auraient pas pu être atteints autrement, enrichissant ainsi potentiellement le processus d'analyse et de supervision. De M'Uzan et d'autres auteurs pensent que l'analyste doit émerger de cet état d'aliénation temporaire et utiliser l'expérience pour représenter des phénomènes qui n’avaient pas encore été symbolisés. 
T. Ogden (1994) s'est indirectement intéressé à cette question d'identité dans le processus analytique. Son concept de «tiers analytique» repose sur le mécanisme d'identification projective. T. Ogden conçoit ce processus comme une tolérance au flou temporaire des limites identitaires permettant d'accueillir, pour ainsi dire, «l'autre»: «L'aspect interpersonnel de l'identification projective implique une transformation de la subjectivité du "contenant" de sorte que le "Je" de l'autre-en-tant-que-sujet est troublé (pour un temps et à un certain degré)». À l'instar de l'idée de Michel De M'Uzan selon laquelle l'analyste doit prêter temporairement sa psyché au patient, T. Ogden écrit :

le contenant de l'identification projective participe à la négation de luimême en tant qu'objet séparé, faisant ainsi «de la place psychologique» en lui-même destinée à être occupée par celui qui projette [...] Ce qui est distinctif dans l'identification projective, en tant que forme de relation analytique, est qu'elle a pour effet de subvertir, de troubler profondément l'expérience de l'analyste et de l'analysant en tant que sujets séparés [...] Le processus analytique implique la réappropriation des subjectivités individuelles de l'analyste et de l'analysant qui ont été transformées à travers l'expérience $[. .$.$] Le contenant ne s'identifie pas simplement à l'autre$ (celui qui projette), il devient un autre [...].

\section{c) Mais comment? Les angles morts: un terrain fertile}

Mais à part l'expérience de l'analyse personnelle et la création d'un espace élaboratif, de quelle façon l'expérience de supervision peut-elle aussi contribuer à faciliter la fluidité des frontières identitaires? Tentons de proposer quelques pistes de réflexion à ce sujet.

Explorons d'abord l'une des fonctions essentielles du superviseur, à savoir celle du repérage des zones de surdité, des angles morts, chez le supervisé dans son travail clinique. Cela constitue un travail délicat qui demande une sensibilité et un courage de la part de deux participants.

Une supervision attentive à la fluidité des frontières identitaires permettra de s'approcher de ce qui se joue dans l'expérience du contre-transfert, et permettre au supervisé de prendre conscience de l'angoisse qui l'habite, voire de ses transferts, projections et résistances mobilisés par une cure en particulier. Le supervisé pourra donc arriver à la perception douloureuse de butées, de répétitions ou de points d'aveuglement du contre-transfert, où il se confrontera à des moments de vide associatif, de sentiment de stagnation ou 
d'impuissance. Le superviseur ne doit surtout pas ignorer ces lieux psychiques qui se présentent durant la rencontre en supervision, car ce sont des lieux qui recèlent un grand potentiel. L'exploration de ces réalités permettra progressivement de deviner l'agissement du transfert, ou, comme Beetschen (2008, p. 36) le nomme, «les zones d'activité du transfert inconscient du patient». La tâche du superviseur sera de tracer, de contenir et de révéler l'impact des forces inconscientes que le supervisé n'a pas pu identifier et élaborer.

Une autre vignette va en ce sens: Laura parle à son superviseur du cas d'une femme ayant des traits obsessionnels et narcissiques très prononcés. Durant les rencontres de supervision, le superviseur est troublé par la difficulté de Laura à reconnaître les gestes d'hostilité implicites dans des remarques sarcastiques et dans des gestes passifs-agressifs subtils que sa patiente lui adressait. Laura et son superviseur ont travaillé à identifier qu'il s'agissait pour Laura d'un angle mort: elle a de la difficulté à constater la charge agressive agissant dans le transfert. Laura prend alors le risque d'associer sur cette difficulté. Peu de temps après ce travail, Laura prend des vacances. Dans la première séance qui suit ces vacances, la patiente, qui avait souffert d'angoisses très fortes durant son absence, attaque Laura d'une manière virulente: elle la rend responsable de toute sa souffrance. Elle menace de se suicider. Laura est paralysée et ressent un vertige; elle est envahie par le sentiment d'être vraiment une mauvaise personne. Elle se contient toutefois, parvient à rester calme et n'intervient presque pas durant la séance. Elle se sentait mal à l'aise à l'idée de venir à sa rencontre de supervision, car elle était terrifiée d'avoir échoué avec cette patiente dans le travail en général, et en particulier pendant la séance rapportée. Le superviseur lui donne son impression: elle était dans une situation extrêmement difficile. Le superviseur constate sa bonne capacité de contention et de tolérance en rapport à l'état de non-savoir, et ce, sans avoir recours à l'action. Laura se met à pleurer de soulagement: elle attendait une critique de la part du superviseur. En élaborant autour de l'expérience avec sa patiente et avec son superviseur, Laura reprend l'exploration et le travail sur cette "zone de surdité», à savoir l'aveuglement à reconnaitre des aspects destructifs dans la dynamique transférentielle-contretransférentielle. Cette difficulté avait un lien aussi avec sa crainte d'une réaction hostile de la part du superviseur; comme si lui aussi allait l'attaquer. Laura et son superviseur ont mis quelques rencontres de supervision à élaborer cette expérience. Laura a risqué une ouverture dans l'espace de supervision qui lui aura finalement permis de faire un travail psychique sur les enjeux liés à la violence du sexuel infantile sous la forme d'une haine envers l'objet. Ils ont reconnu que, 
même si cette expérience avait été très difficile, elle fut très importante autant dans le travail avec la patiente que dans un travail personnel permettant de toucher à nouveau un aspect central de sa conflictualité psychique et ainsi de continuer un travail personnel.

Cette vignette illustre deux points: le premier a trait à ce que Gantheret (2005, p. 265) affirme que «les moments les plus vivants et féconds dans la supervision et en particulier dans l'interaction entre superviseur et supervisé sont quand on peut s'apercevoir de l'actualisation d'une problématique latente, inaperçue et active dans l'analyse elle-même». Elle illustre aussi comment la transmission se construit sur la capacité de contenir les angoisses inconscientes. En nous inspirant du modèle contenant-contenu de Bion qui fut par la suite développé par Ungar et al. (2001), nous pouvons affirmer qu'une des fonctions centrales de la supervision est d'aider à contenir l'agitation émotionnelle et les angoisses qui surgissent dans les deux espaces de travail: l'espace du travail analytique entre le supervisé et son patient et l'espace de travail de supervision entre le superviseur et le supervisé. Il s'agit de deux champs analytiques qui comportent chacun leur propre charge affective issue du rapport et des forces pulsionnelles dans les deux dyades.

Nous voulons soulever à cet effet un point qui nous paraît essentiel quant à la résistance de l'analyste lui-même envers la psychanalyse. Nous sommes d'accord avec Nathalie Zaltzman (2008, p. 60) lorsqu'elle souligne l'importance d'une écoute favorisant un échange élaboratif, mais qui tient compte des «modes d'investissement de la psychanalyse, ses présupposés théoriques explicites, latents, voire inconscients, ses méfiances, ses inquiétudes, les indices transférentiels de sa relation au superviseur». Nous trouvons ce point particulièrement essentiel en ce qui concerne la transmission de la psychanalyse. Il ne s'agit pas seulement d'être attentif aux enjeux du travail analytique, mais aussi d'identifier les modes d'investissement de la psychanalyse. Cela est en lien étroit avec la solidité de l'identité psychanalytique qui sera forgée. Si on n'identifie ni n'élabore les méfiances, les inquiétudes et l'ambivalence que l'on éprouve envers la psychanalyse, il y aura une plus grande difficulté à soutenir l'identité d'analyste, à la transmettre et à l'offrir avec passion et conviction.

L'exploration de ces zones a un point en commun avec le travail d'analyse. Nous élaborerons sur ce sujet dans une autre section de ce texte, portant sur la création de moments d'ouverture où il devient possible de deviner l'agissement des forces inconscientes; moments clés dans les deux champs 
de transmission, où quelque chose du non représenté peut s'introduire dans le domaine psychique; moments d'éclaircissements accompagnés du plaisir associé au lever temporaire du refoulement. La capacité de s'ouvrir à l'expérience en supervision, tel que nous le proposons, a le potentiel de favoriser des changements internes dans l'économie psychique du supervisé, lui permettant ainsi de développer une disponibilité mobile par rapport aux différentes problématiques qu'il rencontrera dans sa pratique.

\section{d) Un regard en surplomb du couple supervisé-patient}

T. Ogden (2005), en suivant Bion, se réfère au travail d'élaboration du supervisé comme étant un travail de «rêve». Le supervisé apportera dans l'espace de supervision la part de son expérience avec son patient qui n'a pas pu être «rêvée» par lui. Pour cet auteur, la difficulté à «rêver» est la conséquence de la turbulence de l'expérience émotionnelle du supervisé avec son patient. Ce fracas émotionnel risque d'avoir eu un effet déstabilisant et d'avoir compromis sa capacité à élaborer l'expérience.

Cette vision du travail de supervision s'ajoute au travail d'identification des angles morts que nous avons abordé plus haut. Elle souligne la présence de la véritable capacité du supervisé à faire un travail psychique en utilisant son écoute associative, sa capacité de rêverie et sa capacité de tenir compte de l'atmosphère dans le champ analytique. Le superviseur interviendra là où cette capacité est perturbée par les émotions et les pensées à caractère dérangeant qui se génèrent inévitablement dans le champ analytique. Il ne s'agit donc pas exclusivement d'identifier les angles morts, mais aussi d'identifier les moments où la capacité d'élaborer, voire de rêver, se trouve perturbée. Le défi du superviseur sera d'identifier ces moments et de promouvoir un travail conjoint avec le supervisé, d'une manière telle que ce dernier puisse retrouver sa position d'analyste.

\section{L'entrecroisement de la supervision et de l'analyse personnelle}

La réflexion que nous venons de proposer sur les complexités et diversités des voies de la transmission laisse entrevoir l'entrecroisement entre l'expérience de l'analyse personnelle et la supervision.

Il existe un paradoxe inhérent à la situation de la supervision: elle relève à la fois de l'analyse quand elle rencontre la scène inconsciente chez chacun de ses protagonistes, et elle ne peut pas être une véritable analyse. C'est donc une tâche importante des deux protagonistes que de s'engager dans un 
travail psychique qui permettra d'élaborer les expériences qui émergent de l'entrecroisement de la relation entre le superviseur et le supervisé et entre le supervisé et son patient.

La fluidité identitaire de la rencontre de supervision permet le surgissement de certaines expériences inattendues. Les phénomènes des processus de reflet décrits par Searles (1955, p. 159) en sont un exemple. Cet auteur a souligné que

les émotions vécues par un superviseur, y compris ses fantasmes subjectifs et privés et ses sentiments personnels envers le supervisé, fournissent souvent des éclaircissements précieux des processus inconscients interpersonnels qui caractérisent la relation actuelle entre le supervisé et son patient. En outre, ces processus sont souvent ceux qui causent des difficultés dans la relation thérapeutique. Les processus inconscients et conscients à l'œuvre dans la relation actuelle entre le patient et le supervisé se reflètent souvent dans la relation consciente et inconsciente entre superviseur et supervisé. Je vais appeler ce phénomène: le «processus de reflet». (Traduction de l'auteure.)

Ce processus est généralement appelé "processus parallèle».

En voici un exemple: Robert présente en supervision le cas d'une femme avec une sensibilité extrême à la critique, de nature paranoïde. Robert est très prudent et évite d'intervenir de peur de provoquer une réaction négative chez sa patiente. Durant cette période, l'atmosphère de l'espace de supervision acquiert un caractère tendu: le superviseur réalise que ses interventions créent une réaction défensive chez Robert, qui se sent mal à l'aise et a eu tendance à inhiber la supervision. Dans un premier temps, le superviseur rationalise ce retrait en se justifiant: il ne veut pas créer une mésalliance avec Robert, il se dit qu'il doit attendre le bon moment, etc. Cependant, le climat continue d'être tendu et le superviseur sent qu'il ne contribue pas de façon positive au processus de supervision. Il décide alors de se laisser intervenir de façon plus spontanée et fait un commentaire concernant une intervention que Robert a faite à son patient. Cela provoque une réaction de colère de Robert, qui se sent semble-t-il profondément critiqué. Le superviseur et Robert ont mis du temps à réfléchir sur ce qui s'était passé dans l'espace de supervision. Quelques rencontres plus tard, Robert dit au superviseur qu'il croyait que sa colère envers lui reflétait la colère qu'il craignait éprouver de sa patiente. Le superviseur a reconnu que ce style défensif chez Robert limitait sa capacité à le contenir. Cet insight a permis d'identifier un angle mort 
qui rendait Robert plus vulnérable à ce type de problématique et qui limitait son travail avec sa patiente.

Très proche de cette perspective, Beetschen (2008) ajoute que la situation de supervision a comme horizon la capacité pour les analystes de rester, dans leurs échanges, des analysants. Le besoin de «raconter» un cas n'obéit pas seulement à des motifs objectifs de clarifier des questions du travail clinique; le choix du cas est souvent lié aux difficultés émotionnelles du supervisé. En ce sens, la rencontre de supervision a le potentiel de prolonger le chemin d'une découverte personnelle.

Tout en considérant ces points d'entrecroisement entre la supervision et l'analyse, il me semble important de souligner que le superviseur et le supervisé doivent conserver la liberté de décider l'importance d'explorer ces dimensions. Chaque expérience de supervision est unique et la flexibilité à offrir un espace adapté à la réalité de chaque supervisé est essentielle.

\section{Les traces de la transmission}

Pour terminer, nous aborderons la question des traces de la transmission. Mais, avant d'examiner ce sujet, nous aimerions dire un mot sur la question de l'idéalisation, aspect qui a le potentiel d'entraver la transmission. Ce thème pourrait certes faire l'objet d'une réflexion plus large, mais nous parlerons seulement ici du fait crucial qu'une certaine idéalisation du superviseur, de l'analyste, et/ou de théorie, est inévitable. Ce qui représente un vrai danger est lorsque l'idéalisation est renforcée par le superviseur ou l'analyste. L. Monette et J. Mauger (2010) nous rappellent que «la psychanalyse court le risque d'être chargée d'une image idéale dont il aura d'autant plus de peine à se défaire qu'elle rejoint la tentation idéalisante de l'analyste lui-même de se laisser prendre pour un autre». A. Lussier (1992) était préoccupé par ces dangers et nous en parlait souvent. Ainsi nous confiait-il qu'il faut éviter d'être la proie de l'idéalisation, ce qui est souvent inévitable. Il a beaucoup insisté sur l'importance pour les analystes de se livrer à une participation ouverte soit au sein d'une supervision, de réunions scientifiques, de congrès etc. et où l'on participe spontanément. Cela veut dire qu'on doit se départir de la tentation narcissique d'être vu comme «le meilleur» et participer à lever cette entrave.

Nous voulons maintenant proposer une réflexion sur les frayages rendus possibles par l'expérience de la transmission.

Il serait souhaitable que l'expérience de supervision agisse de manière à favoriser des changements significatifs dans l'économie psychique du 
supervisé: une capacité accrue du sujet «d'entendre» ses propres zones de surdité, de soutenir, comme le dit M. de M'Uzan, une «inquiétude permanente», une recherche incessante, une remise en question. Ces changements lui permettraient de repérer ses points de résistance - et cela implique aussi les points de résistance envers la psychanalyse elle-même. Il pourrait ainsi développer une disponibilité mobile par rapport aux différentes problématiques qu'il rencontrera dans sa pratique.

Sur le plan de la théorie, on voudrait voir mis en route un mouvement d'exploration continuelle de la validité de la théorie psychanalytique, comme N. Zaltzman (2008, p. 62) l'affirme: «une confrontation incessante entre le savoir et les faits cliniques» On souhaite que le supervisé introjecte la fonction supervisante, qui consiste en la disposition psychique du superviseur qui exerce constamment un regard et une écoute dans sa fonction tiercisante. De par sa manière de penser, le superviseur exprime ce que cela signifie pour lui d'être analyste. Il révèle, comme dirait T. Ogden (2005), «sa capacité de rêver » les rencontres de supervision elles-mêmes. Le superviseur témoigne de la façon dont l'analyste conçoit et exprime ses idées et ses émotions. Il témoigne également en répondant au supervisé en tant qu'individu unique, en reconnaissant sa propre personnalité, qui doit être considérée comme un instrument du travail analytique.

C'est cette disposition qui doit progressivement s'instaurer chez le supervisé. En élaborant cette notion d'introjection de la fonction supervisante, Gantheret (2005, p. 265) fait une distinction importante entre l'incorporation et l'introjection:

Si l'incorporation réside dans la sauvegarde interne de l'objet, par son installation mortifiée et mortifiante dans le sujet, avec tous les effets de mimétisme, entre autres, qu'on peut en attendre - car elle fonctionne d'abord en termes, à peine métaphorisés, de corps, de voix, de posture et de vêture -, l'introjection suppose au contraire un deuil de l'objet et l'appropriation du processus langagier qui en pallie l'absence [...] L'introjection est appropriation d'un processus, non d'un objet à l'inverse de l'incorporation qui est mise en soi de l'objet ou de parties de l'objet, l'introjection est intériorisation vivifiante du pulsionnel de l'autre.

La notion d'espace potentiel, l'espace de jeu développé par Winnicott (1971), ajoute à cette notion d'introjection. Si un objectif de la supervision est d'aider l'analyste en formation à accéder à une plus grande liberté 
de pensée et à construire sa propre identité comme analyste, un espace de jeu est essentiel à ce processus. Winnicott nous rappelle que la présence de l'autre à travers un lien, réel ou imaginé, est nécessaire pour le développement des activités créatrices et ludiques. L'enfant et l'adulte jouent dans un rapport avec l'autre, qu'il s'agisse d'un autre enfant, d'un adulte ou d'une partie de lui-même. L'activité ludique suppose donc autrui; comme témoin, comme compagnon de jeu, comme garant. Cet autrui deviendra, dans le cas de la transmission, le «je», le sujet lui-même, de sorte qu'une introjection de la capacité de jouer pourra être acquise - la capacité de jouer, mais aussi d'être seul.

\section{Gabriela Legorreta mariagabrielalegorreta@gmail.com}

\section{Références}

Baranger,W.(1979). Proceso en espiral y campo dinámico. Revista Uruguaya de Psicoaonálisis, 54, 15-32.

Baranger, M., Baranger, W. et Mom, J. (1983). Process and non-process in analytic work. International Journal of Psychoanalysis, 64, 1-15.

Beetschen, A. (2008). Sur le maniement de la supervision. Topique, 103 (2), 31-40.

Bion, W. (1990). L'attention et l'interprétation. Paris: Payot.

Blanchot, M. (1969). L'entretien infini. Paris: Gallimard.

Brown, L. (2011). Intersubjective Processes and the Unconscious: An Integration of Freudian, Kleinian and Bionian Perspectives. London: New Library of Psychoanalysis.

De Mijolla-Mellor, S. (2008). L'analyse quatrième. Topique, 2 (103), 5-5.

De M’Uzan, M. (2009). L'inquiétante étrangeté ou «Je ne suis pas celle que vous croyez».

Dans L. Danon-Boileau (dir.) Inquiétante étrangeté, p. 89-98. Paris: Presses universitaires de France.

Faimberg, H. (2005). The Telescoping of Generations. London: Routledge.

Filho, G.V. et Pires, A. C. (2010). Benign and Disruptive Disturbances of the Supervisory Field. International Journal of Psychoanalysis, 91 (4), 895-913.

Ferro, A. et Civitarese, G. (2014). The Analaytic Field and its Transformations. London: Karnac. Freud, S. (1923). Conseils aux médecins sur le traitement analytique. Dans S. Freud (1997), La technique psychanalytique (p. 61-71). Paris: Presses universitaires de France.

Gantheret, F. (2005). La représentation est terminée. Penser/Rêver, 8, 259-267.

Lussier, A. (1992). Notre idéologie de formation. Revue française de psychanalyse, 56 (2), 483-487.

Monette, L. et Mauger, J. (2000). L'idéal transmis. Revue française de psychanalyse, 64 (5), 1391-1461.

M’Uzan M. de (1994). La bouche de l'inconscient. Paris: Gallimard.

M’Uzan, M. de (2015). L'inquiétude permanente. Paris: Gallimard.

Ogden, H. T. (1994). The analytic third: Working with intersubjective clinical facts. International Journal of Psychoanalysis, 75 (1), 3-20.

Ogden, H. T. (2005). On psychoanalytic supervision. International Journal of Psychoanalysis, 86 (5), 1265-1280. 
Pontalis, J.-B. (1997). Ce temps qui ne passe pas. Paris: Gallimard.

Robertson, B., Lester, E. P. et Robertson, B. M. (1995). Multiple Interactive Processes in Psychoanalytic Supervision. Psychoanalytic Inquiry, 15 (2), 211-225.

Searles, H. (1955). The informational value of the supervisor's emotional experience. Dans H. Searles (1965), Collected Papers on Schizophrenia and Related Subjects (p. 135-146). New York: International Universities Press.

Scarfone, D. (2014). L'impassé, actualité de l'inconscient. Revue française de psychanalyse, 78 (5), 1357-1428.

Winnicott, D. W. (1971). Playing and reality. New York: Basic Books.

Winnicott, D. W. (1987). The Spontaneous Gesture. Selected letters of D.W. Winnicott. London: Harvard University Press.

Ungar, V. et Ahamada, L. (2001). Supervision: A Container-Contained Approach. International Journal of Psychoanalalysis, 82 (1), 71-81.

Zaltzman, N. (1998). De la guérison psychanalytique. Paris: Presses universitaires de France.

Zaltzman, N. (2001). La mort dans l'âme. Topique, 74 (1), 57-68.

Zaltzman, N. (2008). Entre modèle et fiction, ce que je dois à l'analyse quatrième comme théorie du contrôle. Topique, 103 (2), 9-64. 